\title{
Localization of Ringed Spaces
}

\author{
William D. Gillam \\ Department of Mathematics, Brown University, Providence, USA \\ E-mail:wgillam@math.brown.edu
}

Received March 28, 2011; revised April 12, 2011; accepted April 25, 2011

\begin{abstract}
Let $X$ be a ringed space together with the data $M$ of a set $M_{X}$ of prime ideals of $\mathcal{O}_{X, X}$ for each point $x \in X$. We introduce the localization of $(X, M)$, which is a locally ringed space $Y$ and a map of ringed spaces $Y \rightarrow X$ enjoying a universal property similar to the localization of a ring at a prime ideal. We use this to prove that the category of locally ringed spaces has all inverse limits, to compare them to the inverse limit in ringed spaces, and to construct a very general Spec functor. We conclude with a discussion of relative schemes.
\end{abstract}

Keywords: Localization, Fibered Product, Spec, Relative Scheme

\section{Introduction}

Let Top, LRS, RS, and Sch denote the categories of topological spaces, locally ringed spaces, ringed spaces, and schemes, respectively. Consider maps of schemes $f_{i}: X_{i} \rightarrow Y(i=1,2)$ and their fibered product $X_{1} \times_{Y} X_{2}$ as schemes. Let $\underline{X}$ denote the topological space underlying a scheme $X$. There is a natural comparison map

$$
\eta: \underline{X_{1} \times_{Y} X_{2}} \rightarrow \underline{X}_{1} \times_{\underline{Y}} \underline{X}_{2}
$$

which is not generally an isomorphism, even if $X_{1}, X_{2}, Y$ are spectra of fields (e.g. if $Y=\operatorname{Spec} \mathbb{R}$, $X_{1}=X_{2}=\operatorname{Spec} \mathbb{C}$, the map $\eta$ is two points mapping to one point). However, in some sense $\eta$ fails to be an isomorphism only to the extent to which it failed in the case of spectra of fields: According to [EGA I.3.4.7] the fiber $\eta^{-1}\left(x_{1}, x_{2}\right)$ over a point $\left(x_{1}, x_{2}\right) \in \underline{X}_{1} \times_{\underline{Y}} \underline{X}_{2}$ (with common image $y=f_{1}\left(x_{1}\right)=f_{2}\left(x_{2}\right)$ ) is naturally bijective with the set

$$
\underline{\operatorname{Spec}} k\left(x_{1}\right) \otimes_{k(y)} k\left(x_{2}\right) .
$$

In fact, one can show that this bijection is a homeomorphism when $\eta^{-1}\left(x_{1}, x_{2}\right)$ is given the topology it inherits from $X_{1} \times_{Y} X_{2}$. One can even describe the sheaf of rings $\eta^{-1}\left(x_{1}, x_{2}\right)$ inherits from $X_{1} \times_{Y} X_{2}$ as follows: Let

$$
\begin{aligned}
S\left(x_{1}, x_{2}\right): & =\left\{z \in \operatorname{Spec} \mathcal{O}_{X_{1}, x_{1}} \otimes_{\mathcal{O}_{Y, y}} \mathcal{O}_{X_{2}, x_{2}}: z \mathcal{O}_{X_{i}, x_{i}}\right. \\
& \left.=\mathcal{O}_{x_{i}} \text { for } i=1,2\right\} .
\end{aligned}
$$

Then (Spec of) the natural surjection

$$
\mathcal{O}_{X_{1}, x_{1}} \otimes_{\mathcal{O}_{Y, y}} \mathcal{O}_{X_{2}, x_{2}} \rightarrow k\left(x_{1}\right) \otimes_{k(y)} k\left(x_{2}\right)
$$

identifies Spec $k\left(x_{1}\right) \otimes_{k(y)} k\left(x_{2}\right)$ with a closed subspace of Spec $\overline{\mathcal{O}_{X_{1}, x_{1}}} \otimes_{\mathcal{O}_{Y, y}} \mathcal{O}_{X_{2}, x_{2}}$ and $\mathcal{O}_{X_{1} \times X_{2}} \mid \eta^{-1}\left(x_{1}, x_{2}\right)$ naturally coincides, under the EGA isomorphism, to the restriction of the structure sheaf of $\operatorname{Spec} \mathcal{O}_{X_{1}, x_{1}} \otimes_{\mathcal{O}_{Y, y}} \mathcal{O}_{X_{2}, x_{2}}$ to the closed subspace

$$
\underline{\operatorname{Spec}} k\left(x_{1}\right) \otimes_{k(y)} k\left(x_{2}\right) \subseteq \underline{\operatorname{Spec}_{X_{1}, x_{1}}} \otimes_{\mathcal{O}_{Y, y}} \mathcal{O}_{X_{2}, x_{2}} \cdot{ }^{1}
$$

It is perhaps less well-known that this entire discussion remains true for LRS morphisms $f_{1}, f_{2}$.

From the discussion above, we see that it is possible to describe $X_{1} \times_{Y} X_{2}$, at least as a set, from the following data:

1) the ringed space fibered product $X_{1} \times{ }_{Y}^{\mathrm{RS}} X_{2}$ (which carries the data of the rings $\mathcal{O}_{X_{1}, x_{1}} \otimes_{\mathcal{O}_{Y, y}} \mathcal{O}_{X_{2}, x_{2}}$ as stalks of its structure sheaf) and

2) the subsets $S\left(x_{1}, x_{2}\right) \subseteq \operatorname{Spec} \mathcal{O}_{X_{1}, x_{1}} \otimes_{\mathcal{O}_{Y, y}} \mathcal{O}_{X_{2}, x_{2}}$

It turns out that one can actually recover $X_{1} \times_{Y} X_{2}$ as a scheme solely from this data, as follows: Given a pair $(X, M)$ consisting of a ringed space $X$ and a subset $M_{X} \subseteq \operatorname{Spec} \mathcal{O}_{X, x}$ for each $x \in X$, one can construct a locally ringed space $(X, M)^{\text {loc }}$ with a map of ringed spaces $(X, M)^{l o c} \rightarrow X$. In a special case, this construction coincides with M. Hakim's spectrum of a ringed topos. Performing this general construction to

There is no sense in which this sheaf of rings on Spec $k\left(x_{1}\right) \otimes_{k(y)} k\left(x_{2}\right)$ is “quasi-coherent". It isn't even a module over the usual structure sheaf of $\operatorname{Spec} k\left(x_{1}\right) \otimes_{k(y)} k\left(x_{2}\right)$ 


$$
\left(X_{1} \times_{Y}^{\mathrm{RS}} X_{2},\left\{S\left(x_{1}, x_{2}\right)\right\}\right)
$$

yields the comparison map $\eta$, and, in particular, the scheme $X_{1} \times_{Y} X_{2}$. A similar construction in fact yields all inverse limits in LRS ( $\$ 3.1)$ and the comparison map to the inverse limit in RS, and allows one to easily prove that a finite inverse limits of schemes, taken in LRS, is a scheme (Theorem 8). Using this description of the comparison map $\eta$ one can easily describe some circumstances under which it is an isomorphism $(\S 3.2)$, and one can easily see, for example, that it is a localization morphism (Definition 1), hence has zero cotangent complex.

The localization construction also allows us construct ( 3.3 ), for any $X \in \mathbf{L R S}$, a very general relative spec functor

$$
\operatorname{Spec}_{X}:\left(\mathcal{O}_{X}-\mathbf{A l g}\right)^{\text {op }} \rightarrow \text { LRS } / X
$$

which coincides with the usual one when $X$ is a scheme and we restrict to quasi-coherent $\mathcal{O}_{X}$ algebras. We can also construct ( $\$ 3.5)$ a "good geometric realization" functor from M. Hakim's stack of relative schemes over a locally ringed space $X$ to $\mathbf{L R S} / X .^{2}$ It should be emphasized at this point that there is essentially only one construction, the localization of a ringed space of $\S 2.2$, in this paper, and one (fairly easy) theorem (Theorem 2) about it; everything else follows formally from general nonsense.

Despite all these results about inverse limits, I stumbled upon this construction while studying direct limits. I was interested in comparing the quotient of, say, a finite étale groupoid in schemes, taken in sheaves on the étale site, with the same quotient taken in LRS. In order to compare these meaningfully, one must somehow put them in the same category. An appealing way to do this is to prove that the (functor of points of the) LRS quotient is a sheaf on the étale site. In fact, one can prove that for any $X \in \mathbf{L R S}$, the presheaf

$$
Y \mapsto \operatorname{Hom}_{\mathrm{LRS}}(Y, X)
$$

is a sheaf on schemes in both the fppf and fpqc topologies. Indeed, one can easily describe a topology on RS, analogous to the fppf and fpqc topologies on schemes, and prove it is subcanonical. To upgrade this to a subcanonical topology on LRS one is naturally confronted with the comparison of fibered products in LRS and RS . In particular, one is confronted with the question of whether $\eta$ is an epimorphism in the category of ringed spaces. I do not know whether this is true for arbitrary LRS morphisms $f_{1}, f_{2}$, but in the case of schemes it is possible to prove a result along these lines which is suf-

$\overline{{ }^{2} \text { Hakim already constructed such a functor, but ours is different from }}$ hers. ficient to upgrade descent theorems for $\mathbf{R S}$ to descent theorems for Sch.

\section{Localization}

We will begin the localization construction after making a few definitions.

Definition 1. A morphism $f: A \rightarrow B$ of sheaves of rings on a space $X$ is called a localization morphism ${ }^{3}$ iff there is a multiplicative subsheaf $S \subseteq A$ so that $f$ is isomorphic to the localization $A \rightarrow S^{-1} A$ of $A$ at $S .^{4}$ A morphism of ringed spaces $f: X \rightarrow Y$ is called a localization morphism iff $f^{\#}: f^{-1} \mathcal{O}_{Y} \rightarrow \mathcal{O}_{X}$ is a localization morphism.

A localization morphism $A \rightarrow B$ in $\operatorname{Rings}(X)$ is both flat and an epimorphism in $\operatorname{Rings}(X){ }^{5}$ In particular, the cotangent complex (hence also the sheaf of Kähler differentials) of a localization morphism is zero [Ill II.2.3.2]. The basic example is: For any affine scheme $X=\operatorname{Spec} A, \underline{A}_{X} \rightarrow \mathcal{O}_{X}$ is a localization morphism.

Definition 2. Let $A$ be a ring, $S \subseteq \operatorname{Spec} A$ any subset. We write $\operatorname{Spec}_{A} S$ for the locally ringed space whose underlying topological space is $S$ with the topology it inherits from Spec $A$ and whose sheaf of rings is the inverse image of the structure sheaf of $\operatorname{Spec} A$.

If $A$ is clear from context, we drop the subscript and simply write $\operatorname{Spec} S$. There is one possible point of confusion here: If $I \subseteq A$ is an ideal, and we think of $\operatorname{Spec} A / I$ as a subset of $\operatorname{Spec} A$, then

$$
\operatorname{Spec}_{A}(\operatorname{Spec} A / I) \neq \operatorname{Spec} A / I
$$

(though they have the same topological space).

\subsection{Prime Systems}

Definition 3. Let $X=\left(X, \mathcal{O}_{X}\right)$ be a ringed space. A prime system $M$ on $X$ is a map $x \mapsto M_{x}$ assigning a subset $M_{x} \subseteq \operatorname{Spec} \mathcal{O}_{X, x}$ to each point $x \in X$. For prime systems $M, N$ on $X$ we write $M \subseteq N$ to mean $M_{x} \subseteq N_{x}$ for all $x \in X$. Prime systems on $X$ form a category $\operatorname{PS}(X)$ where there is a unique morphism from $M$ to $N$ iff $M \subseteq N$. The intersection $\cap_{i} M_{i}$ of prime systems $M_{i} \in \mathbf{P S}(X)$ is defined by

$$
\left(\cap_{i} M_{i}\right)_{X}:=\cap_{i}\left(M_{i}\right)_{x} .
$$

A primed ringed space $(X, M)$ is a ringed space $X$ equipped with a prime system $M$. Prime ringed spaces form a category PRS where a morphism $f:(X, M)$ $\rightarrow(Y, N)$ is a morphism of ringed spaces $f$ satisfy-

\footnotetext{
${ }^{3}$ See [Ill II.2.3.2] and the reference therein.

${ }^{4}$ See [III II.2.3.2] and the reference therein.

${ }^{5}$ Both of these conditions can be checked at stalks.
} 
ing

$$
\left(\operatorname{Spec} f_{x}\right)\left(M_{x}\right) \subseteq N_{f(x)}
$$

for every $x \in X$.

The inverse limit of a functor $i \mapsto M_{i}$ to $P S(X)$ is clearly given by $\cap_{i} M_{i}$.

Remark 1. Suppose $(Y, N) \in$ PRS and $f: X \rightarrow Y$ is an RS morphism. The inverse image $f^{*} N$ is the prime system on $X$ defined by

$$
\begin{aligned}
\left(f^{*} N\right)_{X} & :=\left(\operatorname{Spec} f_{x}\right)^{-1}\left(N_{f(x)}\right) \\
& =\left\{\mathcal{O} \in \operatorname{Spec} \mathcal{O}_{X, x}: f_{x}^{-1}(\mathcal{O}) \in N_{f(x)}\right\} .
\end{aligned}
$$

Formation of inverse image prime systems enjoys the expected naturality in $f: g^{*}\left(f^{*} M\right)=(f g)^{*} M$. We can alternatively define a PRS morphism $f:(X, M)$ $\rightarrow(Y, N)$ to be an RS morphism $f: X \rightarrow Y$ such that c $M \subseteq f^{*} N$ (i.e. together with a $\operatorname{PS}(X)$ morphism $\left.M \rightarrow f^{*} N\right)$.

For $X \in \mathbf{L R S}$, the local prime system $\mathcal{M}_{X}$ on $X$ is defined by $\mathcal{M}_{X, X}:=\left\{\mathfrak{m}_{X}\right\}$. If $Y$ is another locally ringed space, then a morphism $f: X \rightarrow Y$ in $\mathbf{R S}$ defines a morphism of primed ringed spaces

$f:\left(X, \mathcal{M}_{X}\right) \rightarrow\left(Y, \mathcal{M}_{Y}\right)$ iff $f$ is a morphism in LRS, so we have a fully faithful functor

$$
\begin{aligned}
& \mathcal{M}: L R S \rightarrow P R S \\
& X \mapsto\left(X, \mathcal{M}_{X}\right),
\end{aligned}
$$

and we may regard LRS as a full subcategory of PRS .

At the "opposite extreme" we also have, for any $X \in \mathbf{R S}$, the terminal prime system $\mathcal{T}_{X}$ defined by $\mathcal{T}_{X, X}:=\operatorname{Spec} \mathcal{O}_{X, X}$ (i.e. the terminal object in $\operatorname{PS}(X)$ ). For $(Y, M) \in \mathbf{P R S}$, we clearly have

$$
\operatorname{Hom}_{\mathbf{P R S}}\left((Y, M),\left(X, \mathcal{T}_{X}\right)\right)=\operatorname{Hom}_{\mathbf{R S}}(Y, X),
$$

so the functor

$$
\begin{aligned}
& \mathcal{T}: \mathbf{R S} \rightarrow \text { PRS } \\
& X \mapsto\left(X, \mathcal{T}_{X}\right)
\end{aligned}
$$

is right adjoint to the forgetful functor $\mathbf{P R S} \rightarrow \mathbf{R S}$ given by $(X, M) \mapsto X$.

\subsection{Localization}

Now we begin the main construction of this section. Let $(X, M)$ be a primed ringed space. We now construct a locally ringed space $(X, M)^{\text {loc }}$ (written $X^{\text {loc }}$ if $M$ is clear from context), and a PRS morphism $\pi:\left(X^{\text {loc }}, \mathcal{M}_{X}\right.$ loc $) \rightarrow(X, M)$ called the localization of

Definition 4. Let $X$ be a topological space, $\mathscr{F}$ a sheaf on $X$. The category Sec $F$ of local sections of is the category whose objects are pairs $(U, s)$ where $U$ is an open subset of $X$ and $s \in \mathscr{F}(U)$, and where there is a unique morphism $(U, s) \rightarrow(V, t)$ if $U \subseteq V$ and $t \mid U=s$.

As a set, the topological space $X^{\text {loc }}$ will be the set of pairs $(x, z)$, where $x \in X$ and $z \in M_{x}$. Let $\mathcal{P}\left(X^{\text {loc }}\right)$ denote the category of subsets of $X^{\text {loc }}$ whose morphisms are inclusions. For $(U, s) \in \operatorname{Sec} \mathcal{O}_{X}$, set

$$
U(U, s):=\left\{(x, z) \in X^{l o c}: x \in U, s_{x} \notin z\right\} .
$$

This defines a functor

satisfying:

$$
U: \operatorname{Sec} \mathcal{O}_{X} \rightarrow \mathcal{O}\left(X^{l o c}\right)
$$

$$
\begin{aligned}
& U(U, s) \cap U(V, t)=U\left(U \cap V,\left.\left.s\right|_{U \cap V} t\right|_{U \cap V}\right) \\
& U\left(U, s^{n}\right)=U(U, s) \quad\left(n \in \mathbb{Z}_{>0}\right) .
\end{aligned}
$$

The first formula implies that $U\left(\operatorname{Sec} \mathcal{O}_{X}\right) \subseteq \mathcal{O}\left(X^{\text {loc }}\right)$ is a basis for a topology on $X^{\text {loc }}$ where a basic open neighborhood of $(x, z)$ is a set $U(U, s)$ where $x \in U$, $s_{X} \notin Z$. We always consider $X^{l o c}$ with this topology. The map

$$
\begin{aligned}
& \pi: X^{l o c} \rightarrow X \\
& (x, z) \mapsto X
\end{aligned}
$$

is continuous because $\pi^{-1}(U)=U(U, 1)$.

We construct a sheaf of rings $\mathcal{O}_{X}$ loc on $X^{\text {loc }}$ as follows. For an open subset $V \subseteq X^{\text {loc }}$, we let $\mathcal{O}_{X}$ loc $(V)$ be the set of

$$
s=(s(x, z)) \in \prod_{(x, z) \in V}\left(\mathcal{O}_{X, x}\right)_{z}
$$

satisfying the local consistency condition: For every $(x, z) \in V$, there is a basic open neighborhood $U(U, t)$ of $(x, z)$ contained in $V$ and a section

$$
\frac{a}{t^{n}} \in \mathcal{O}_{X}(U)_{t}
$$

such that, for every $\left(x^{\prime}, z^{\prime}\right) \in U(U, t)$, we have

$$
s\left(x^{\prime}, z^{\prime}\right)=\frac{a_{x^{\prime}}}{t_{x^{\prime}}^{n}} \in\left(\mathcal{O}_{X, x^{\prime}}\right)_{z^{\prime}} .
$$

(Of course, one can always take $n=1$ since $U(U, t)=U\left(U, t^{n}\right)$.) The set $\mathcal{O}_{X l o c}(V)$ becomes a ring under coordinatewise addition and multiplication, and the obvious restriction maps make $\mathcal{O}_{\text {loc }}$ a sheaf of rings on $X^{\text {loc }}$. There is a natural isomorphism

$$
\mathcal{O}_{X}^{l o c},(x, z)=\left(\mathcal{O}_{X, X}\right)_{z}
$$

taking the germ of $s=(s(x, z)) \in \mathcal{O}_{X \text { loc }}(U)$ in the stalk $\mathcal{O}_{X \text { loc },(x, z)}$ to $s(x, z) \in\left(\mathcal{O}_{X, x}\right)_{z}$. This map is injective because of the local consistency condition and surjective 
because, given any $a / b \in\left(\mathcal{O}_{X, X}\right)$, we can lift $a, b$ to $\bar{a}, \bar{b} \in \mathcal{O}_{X}(U)$ on some neighborhood $U$ of $x$ and define $s \in \mathcal{O}_{X^{\text {loc }}}(U(U, \bar{b}))$ by letting

$s\left(x^{\prime}, z^{\prime}\right):=\bar{a}_{x^{\prime}} / \bar{b}_{x^{\prime}} \in\left(\mathcal{O}_{X, x^{\prime}}\right)_{z^{\prime}}$. This $s$ manifestly satisfies

the local consistency condition and has $s(x, z)=a / b$. In particular, $X^{\text {loc }}$, with this sheaf of rings, is a locally ringed space.

To lift $\pi$ to a map of ringed spaces $\pi: X^{\text {loc }} \rightarrow X$ we use the tautological map

$$
\pi^{\#}: \mathcal{O}_{X} \rightarrow \pi_{*} \mathcal{O}_{X} \text { loc }
$$

of sheaves of rings on $X$ defined on an open set $U \subseteq X$ by

$$
\begin{aligned}
& \pi^{\#}(U): \mathcal{O}_{X}(U) \rightarrow\left(\pi_{*} \mathcal{O}_{X \text { loc }}\right)(U)=\mathcal{O}_{X^{l o c}}(U(U, 1)) \\
& s \mapsto\left(\left(s_{X}\right)_{z}\right) .
\end{aligned}
$$

It is clear that the induced map on stalks

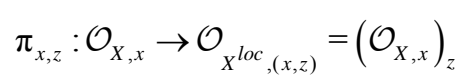

is the natural localization map, so $\pi_{x, z}^{-1}\left(\mathcal{O}_{z}\right)=z \in M_{x}$ and hence $\pi$ defines a PRS morphism

$\pi:\left(X^{l o c}, \mathcal{O}_{X^{l o c}}\right) \rightarrow(X, M)$.

Remark 2. It would have been enough to construct the localization $\left(X, \mathcal{T}_{X}\right)^{\text {loc }}$ at the terminal prime system. Then to construct the localization $(X, M)^{\text {loc }}$ at any other prime system, we just note that $(X, M)^{\text {loc }}$ is clearly a subset of $\left(X, \mathcal{T}_{X}\right)^{\text {loc }}$, and we give it the topology and sheaf of rings it inherits from this inclusion. The construction of $\left(X, \mathcal{T}_{X}\right)^{\text {loc }}$ is "classical." Indeed, M. Hakim [Hak] describes a construction of $\left(X, \mathcal{T}_{X}\right)^{\text {loc }}$ that makes sense for any ringed topos $X$ (she calls it the spectrum of the ringed topos [Hak IV.1]), and attributes the analogous construction for ringed spaces to $\mathrm{C}$. Chevalley [Hak IV.2]. Perhaps the main idea of this work is to define "prime systems," and to demonstate their ubiquity. The additional flexibility afforded by non-terminal prime systems is indispensible in the applications of $\S 3$. It is not clear to me whether this setup generalizes to ringed topoi.

We sum up some basic properties of the localization map $\pi$ below.

Proposition 1. Let $(X, M)$ be a primed ringed space with localization $\pi: X^{\text {loc }} \rightarrow X$. For $x \in X$, the fiber $\pi^{-1}(x)$ is naturally isomorphic in LRS to $\operatorname{Spec} M_{x}$ (Definition 2). ${ }^{6}$ Under this identification, the stalk of $\pi$

${ }^{6} \mathrm{By}$ "fiber" here we mean $\pi^{-1}(x):=X^{\text {loc }} \times_{x}^{\mathrm{RS}}\left(\{x\}, \mathcal{O}_{x, x}\right)$, which is just the set theoretic preimage $\pi^{-1}(x) \subseteq X^{\text {loc }}$ with the topology and sheaf of rings it inherits from $X^{\text {loc }}$. This differs from another common usage of "fiber" to mean $X^{\text {loc }} x_{X}^{\mathrm{RS}}(\{x\}, k(x))$. at $z \in M_{x}$ is identified with the localization of $\mathcal{O}_{X, x}$ at $z$, hence $\pi$ is a localization morphism (Definition 1).

Proof. With the exception of the fiber description, everything in the proposition was noted during the construction of the localization. Clearly there is a natural bijection of sets $M_{x}=\pi^{-1}(x)$ taking $z \in M_{x}$ to $(x, z) \in \pi^{-1}(x)$. We first show that the topology inherited from $X^{\text {loc }}$ coincides with the one inherited from $\operatorname{Spec} \mathcal{O}_{X, X}$. By definition of the topology on $X^{\text {loc }}$, a basic open neighborhood of $z \in M_{X}$ is a set of the form

$$
U(U, s) \cap M_{x}=\left\{z^{\prime} \in M_{x}: s_{x} \notin z^{\prime}\right\},
$$

where $U$ is a neighborhood of $x$ in $X$ and $s \in \mathcal{O}_{X}(U)$ satisfies $s_{X} \notin Z$. Clearly this set depends only on the stalk of $s_{X} \in \mathcal{O}_{X, X}$ of $s$ at $x$, and any element $t \in \mathcal{O}_{X, X}$ lifts to a section $\bar{t} \in \mathcal{O}_{X}(U)$ on some neighborhood of $X$, so the basic neighborhoods of $z \in M_{x}$ are the sets of the form

$$
\left\{z^{\prime} \in M_{x}: t \notin z^{\prime}\right\}
$$

where $t_{x} \notin z$. But for the same set of $t$, the sets

$$
D(t):=\left\{\mathfrak{p} \in \operatorname{Spec} \mathcal{O}_{X, X}: t \notin \mathfrak{p}\right\}
$$

form a basis for neighborhoods of $z$ in $\operatorname{Spec} \mathcal{O}_{X, X}$ so the result is clear.

We next show that the sheaf of rings on $M_{X}$ inherited from $X^{\text {loc }}$ is the same as the one inherited from $\operatorname{Spec} \mathcal{O}_{X, X}$. Given $f \in \mathcal{O}_{X, X}$, a section of $\mathcal{O}_{X \text { loc }} \mid M_{x}$ over the basic open set $M_{X} \cap D(f)$ is an element

$$
s=(s(z)) \in \prod_{z \in M_{X} \cap D(f)}\left(\mathcal{O}_{X, x}\right)_{z}
$$

satisfying the local consistency condition: For all $z \in M_{x} \cap D(f)$, there is a basic open neighborhood $U(U, t)$ of $(x, z)$ in $X^{\text {loc }}$ and an element $a /\left(t^{n}\right) \in \mathcal{O}_{X}(U)_{t}$ such that, for all $z^{\prime} \in M_{x} \cap D(f)^{t} \cap U(U, t)$, we have $s\left(z^{\prime}\right) \in a_{z^{\prime}} /\left(t_{z^{\prime}}^{n}\right)$. Note that

$$
M_{x} \cap D(f) \cap U(U, t)=M_{x} \cap D\left(f t_{x}\right)
$$

and $a_{x} /\left(t_{x}^{n}\right) \in \mathcal{O}_{\text {Spec } \mathcal{O}_{X, x}}\left(D\left(f t_{x}\right)\right)$. The sets $D\left(f t_{x}\right) \cap M_{x}$ cover $M_{x} \cap D(f) \subseteq$ Spec $\mathcal{O}_{X, X}$, and we have a "global formula" $s$ showing that the stalks of the various $a_{x} /\left(t_{x}^{n}\right)$ agree at any $z \in M_{x} \cap D(f)$, so they glue to yield an element $g(s) \in \mathcal{O}_{\mathrm{Spec}_{X, x}}\left(M_{x} \cap D(f)\right)$ with $g(s)_{z}=s(z)$. We can define a morphism of sheaves on $M_{x}$ by defining it on basic opens, so this defines a morphism of sheaves $g: \mathcal{O}_{X \text { loc }}\left|M_{x} \rightarrow \mathcal{O}_{\text {Spec }}\right|_{X, x} \mid M_{x}$ which is easily seen to be an isomorphism on stalks.

Remark 3. Suppose $(X, M) \in \mathbf{P R S}$ and $U \subseteq X$ is an open subspace of $X$. Then it is clear from the construction of $\pi:(X, M)^{\text {loc }} \rightarrow X$ that

$$
\pi^{-1}(U)=\left(U, \mathcal{O}_{X}|U, M| U\right)^{l o c} .
$$


The following theorem describes the universal property of localization.

Theorem 2. Let $f:(X, M) \rightarrow(Y, N)$ be a morphism in PRS. Then there is a unique morphism $\bar{f}:(X, M)^{\text {loc }} \rightarrow(Y, N)^{\text {loc }}$ in LRS making the diagram

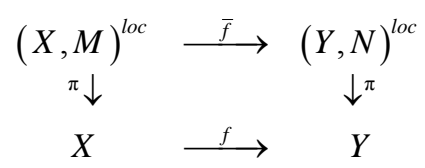

commute in RS . Localization defines a functor

$$
\begin{aligned}
\text { PRS } & \rightarrow \text { LRS } \\
(X, M) & \mapsto(X, M)^{l o c} \\
f:(X, M) \rightarrow(Y, N) & \mapsto \bar{f}:(X, M)^{l o c} \rightarrow(Y, N)^{l o c}
\end{aligned}
$$

retracting the inclusion functor $\mathcal{M}: \mathbf{L R S} \rightarrow$ PRS and right adjoint to it: For any $Y \in \mathbf{L R S}$, there is a natural bijection

$$
\operatorname{Hom}_{\mathrm{LRS}}\left(Y,(X, M)^{\text {loc }}\right)=\operatorname{Hom}_{\mathrm{PRS}}\left(\left(Y, \mathcal{M}_{\mathrm{Y}}\right),(X, M)\right) \text {. }
$$

Proof. We first establish the existence of such a morphism $\bar{f}$. The fact that $f$ is a morphism of primed ringed spaces means that we have a function

$$
\begin{aligned}
M_{x} & \rightarrow N_{f(x)} \\
z & \mapsto f_{x}^{-1}(z)
\end{aligned}
$$

for each $x \in X$, so we can complete the diagram of topological spaces

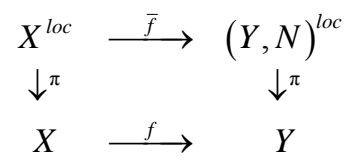

(at least on the level of sets) by setting

$$
\bar{f}(x, z):=\left(f(x), f_{x}^{-1}(z)\right) \in Y^{l o c} .
$$

To see that $\bar{f}$ is continuous it is enough to check that the preimage $\bar{f}^{-1}(U(U, s))$ is open in $X^{\text {loc }}$ for each basic open subset $U(U, s)$ of $Y^{\text {loc }}$. But it is clear from the definitions that

$$
\bar{f}^{-1} U(U, s)=U\left(f^{-1}(U), f^{\#} f^{-1}(s)\right)
$$

(note $\left(f^{\#} f^{-1}(s)\right)=f_{x}\left(s_{f(x)}\right)$ ).

Now we want to define a map $\bar{f}^{\#}: \bar{f}^{-1} \mathcal{O}_{\text {loc }} \rightarrow \mathcal{O}_{Y}$ of sheaves of rings on $Y$ (with "local stalks") making the diagram

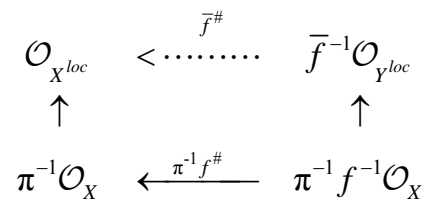

commute in $\operatorname{Rings}\left(Y^{\text {loc }}\right)$. The stalk of this diagram at $(x, z) \in X^{\text {loc }}$ is a diagram

$$
\begin{array}{ccc}
\left(\mathcal{O}_{X, X}\right)_{z} & <\cdots \cdots \cdots & \left(\mathcal{O}_{Y, f(x)}\right) f_{X}^{-1} Z \\
\pi_{(x, z)} \uparrow & & \uparrow^{\pi}\left(f(x), f_{x}^{-1}(z)\right) \\
\mathcal{O}_{X, X} & \longleftarrow & \mathcal{O}_{Y, f(x)}
\end{array}
$$

in Rings where the vertical arrows are the natural localization maps; these are epimorphisms, and the universal property of localization ensures that there is a unique local morphism of local rings $\bar{f}_{(x, 2)}$ completing this diagram. We now want to show that there is actually a (necessarily unique) map $\bar{f}^{\#}: \bar{f}^{-1} \mathcal{O}_{X \text { loc }} \rightarrow \mathcal{O}_{Y}$ of sheaves of rings on $X^{\text {loc }}$ whose stalk at $(x, z)$ is the map $\bar{f}_{(x, z)}$. By the universal property of sheafification, we can work with the presheaf inverse image $\bar{f}_{\text {pre }}^{-1} \mathcal{O}_{x l o c}$ instead. A section $[V, s]$ of this presheaf over an open subset $W \subseteq X^{\text {loc }}$ is represented by a pair $(V, s)$ where $V \subseteq Y^{l o c}$ is an open subset of $Y^{\text {loc }}$ containing $\bar{f}(W)$ and

$$
s=(s(y, z)) \in \mathcal{O}_{Y}{ }_{Y}(V) \subseteq \prod_{(y, z) \in V}\left(\mathcal{O}_{Y, y}\right)_{z} .
$$

I claim that we can define a section $f_{\text {pre }}^{\#}[V, s] \in \mathcal{O}_{X \text { loc }}(W)$ by the formula

$$
f_{\mathrm{pre}}^{\#}[V, s](x, z):=s\left(f(x), f_{x}^{-1}(z)\right) .
$$

It is clear that this element is independent of replacing $V$ with a smaller neighborhood of $f[W]$ and restricting $S$, but we still must check that

$$
f_{\mathrm{pre}}[V, s] \in \prod_{(x, z) \in W}\left(\mathcal{O}_{X, x}\right)_{z}
$$

satisfies the local consistency condition. Suppose

$$
\frac{a}{t^{n}} \in \mathcal{O}_{X}(U)_{t}
$$

witnesses local consistency for $s \in \mathcal{O}_{\text {Yloc }}(V)$ on a basic open subset $U(U, t) \subseteq V$. Then it is straightforward to check that the restriction of

$$
\frac{f^{\#}\left(f^{-1}(a)\right)}{f^{\#}\left(f^{-1} t^{n}\right)} \in \mathcal{O}_{Y}\left(\bar{f}^{-1}(U(U, t))\right)
$$

to $\bar{f}^{-1} U(U, t) \cap W$ witnesses local consistency of $f_{\mathrm{pre}}^{\#}[V, s]$ on

$$
\bar{f}^{-1}(U(U, t)) \cap W=U\left(f^{-1}(U), f^{\#} f^{-1} t\right) \cap W .
$$

It is clear that our formula for $f_{\mathrm{pre}}^{\#}[V, s]$ respects restrictions and has the desired stalks and commutativity, so its sheafification provides the desired map of sheaves of rings.

This completes the construction of $\bar{f}: X^{\text {loc }} \rightarrow Y^{\text {loc }}$ in 
LRS making (3) commute in RS. We now establish the uniqueness of $\bar{f}$. Suppose $\bar{f}^{\prime}: X^{\text {loc }} \rightarrow Y^{\text {loc }}$ is a morphism in LRS that also makes (3) commute in RS . We first prove that $\bar{f}=\bar{f}^{\prime}$ on the level of topological spaces. For $x \in X$ the commutativity of (3) ensures that $\bar{f}^{\prime}(x, z)=\left(f(x), z^{\prime}\right)$ for some $z^{\prime} \in N_{f(x)} \subseteq \operatorname{Spec}_{Y, f(x)}$, so it remains only to show that $z^{\prime}=f_{x}^{-1}(z)$. The commutativity of (3) on the level of stalks at $(x, z) \in X^{\text {loc }}$ gives a commutative diagram of rings

$$
\begin{array}{ccc}
\left(\mathcal{O}_{X, x}\right)_{z} & \stackrel{\bar{f}_{(x, z)}}{\longleftarrow} & \left(\mathcal{O}_{Y, f(x)}\right)_{z} \\
\pi(x, z) \uparrow & \uparrow \pi\left(f(x), z^{\prime}\right) \\
\mathcal{O}_{X, X} & \stackrel{\pi^{-1} f^{\#}}{ } & \mathcal{O}_{Y, f(x)}
\end{array}
$$

where the vertical arrows are the natural localization maps. From the commutativity of this diagram and the fact that $\left(\bar{f}^{\prime}\right)_{(x, z)}^{-1}\left(\mathfrak{m}_{z}\right)=\mathfrak{m}_{z^{\prime}}$ (because $\bar{f}_{(x, z)}^{\prime}$ is local) we find

$$
\begin{aligned}
z^{\prime} & =\pi_{\left(f(x), z^{\prime}\right)}^{-1}\left(\mathfrak{m}_{z^{\prime}}\right) \\
& =\pi_{\left(f(x), z^{\prime}\right)}^{-1}\left(\bar{f}^{\prime}\right)_{(x, z)}^{-1}\left(\mathfrak{m}_{z}\right) \\
& =f_{x}^{-1} \pi_{x}^{-1}\left(\mathfrak{m}_{z}\right) \\
& =f_{x}^{-1}(z)
\end{aligned}
$$

as desired. This proves that $\bar{f}=\bar{f}^{\prime}$ on topological spaces, and we already argued the uniqueness of $\bar{f}^{\#}$ (which can be checked on stalks) during its construction.

The last statements of the theorem follow easily once we prove that the localization morphism

$\pi:\left(X, \mathcal{O}_{X}\right)^{\text {loc }} \rightarrow X$ is an isomorphism for any $X \in$ LRS . On the level of topological spaces, it is clear that $\pi$ is a continuous bijection, so to prove it is an isomorphism we just need to prove it is open. To prove this, it is enough to prove that for any $(U, s) \in \operatorname{Sec} \mathcal{O}_{X}$, the image of the basic open set $U(U, s)$ under $\pi$ is open in $X$. Indeed,

$$
\begin{aligned}
\pi(U(U, s)) & =\left\{x \in U: s_{x} \notin \mathcal{O}_{x}\right\} \\
& =\left\{x \in U: s_{x} \in \mathcal{O}_{X, x}^{*}\right\}
\end{aligned}
$$

is open in $U$, hence in $X$, because invertibility at the stalk implies invertibility on a neighborhood. To prove that $\pi$ is an isomorpism of locally ringed spaces, it remains only to prove that $\pi^{\#}: \mathcal{O}_{X} \rightarrow \mathcal{O}_{X}$ loc is an isomorphism of sheaves of rings on $X=X^{\text {loc }} X^{\text {loc }}$. Indeed, Proposition 1 says the stalk of $\pi^{\#}$ at $\left(x, \mathfrak{m}_{x}\right) \in X^{\text {loc }}$ is the localization of the local ring $\mathcal{O}_{X, X}$ at its unique maximal ideal, which is an isomorphism in LAn .

Lemma 3. Let $A \in$ Rings be a ring, $\left(X, \mathcal{O}_{X}\right):=\operatorname{Spec} A$, and let * be the punctual space.
Define a prime system $N$ on $\left(X, \underline{A}_{X}\right)$ by

$$
N_{X}:=\{x\} \subseteq \operatorname{Spec} \underline{A}_{X, X}=\operatorname{Spec} A=X .
$$

Let $a:\left(X, \mathcal{O}_{X}\right) \rightarrow\left(X, \underline{A}_{X}\right)$ be the natural RS morphism. Then $\mathcal{M}_{\left(X, \mathcal{O}_{X}\right)}=a^{*} N$ and the natural PRS morphisms

$$
\begin{aligned}
& \left(X, \mathcal{O}_{X}, \mathcal{O}_{\left(X, \mathcal{O}_{X}\right)}\right) \rightarrow\left(X, \underline{A}_{X}, N\right) \rightarrow(*, A, \operatorname{Spec} A) \\
& =\left(*, A, \mathcal{T}_{(*, A)}\right)
\end{aligned}
$$

yield natural isomorphisms

$$
\begin{aligned}
\left(X, \mathcal{O}_{X}\right) & =\left(X, \mathcal{O}_{X}, \mathcal{O}_{\left(X, \mathcal{O}_{X}\right)}\right)^{\text {loc }}=\left(X, \underline{A}_{X}, N\right)^{\text {loc }} \\
& =\left({ }^{*}, A, \operatorname{Spec} A\right)^{\text {loc }}
\end{aligned}
$$

in LRS .

Proof. Note that the stalk $a_{x}: \underline{A}_{X, x} \rightarrow \mathcal{O}_{X, x}$ of $a$ at $x \in X$ is the localization map $A \rightarrow A_{x}$, and, by definition, $\left(a^{*} N\right)_{x}$ is the set prime ideals $z$ of $A_{x}$ pulling back to $x \subseteq A$ under $a_{x}: A \rightarrow A_{x}$. The only such prime ideal is the maximal ideal $\mathfrak{m}_{x} \subseteq A_{x}$, so

$$
\left(a^{*} N\right)_{x}=\left\{\mathfrak{m}_{x}\right\}=\mathcal{M}_{\left(X, \mathcal{O}_{X}\right), x} .
$$

Next, it is clear from the description of the localization of a PRS morphism that the localizations of the morphisms in question are bijective on the level of sets. Indeed, the bijections are given by

$$
x \leftrightarrow\left(x, \mathfrak{m}_{x}\right) \leftrightarrow(x, x) \leftrightarrow(*, x),
$$

so to prove that they are continuous, we just need to prove that they have the same topology. Indeed, we will show that they all have the usual (Zariski) topology on $X=\operatorname{Spec} A$. This is clear for $\left(X, \mathcal{O}_{X}, \mathcal{M}_{\left(X, \mathcal{O}_{X}\right)}\right)$ because localization retracts $\mathcal{M}$ (Theorem 2), so $\left(X, \mathcal{O}_{X}, \mathcal{M}_{\left(X, \mathcal{O}_{X}\right)}\right)^{\text {loc }}=\left(X, \mathcal{O}_{X}\right)$, and it is clear for

$(*, A, \operatorname{Spec} A)$ because of the description of the fibers of localization in Proposition 1. For $\left(X, \underline{A}_{X}, N\right)$, we note that the sets $U(U, s)$, as $U$ ranges over connected open subsets of $X$ (or any other family of basic opens for that matter), form a basis for the topology on $\left(X, \underline{A}_{X}, N\right)^{l o c}$. Since $U$ is connected, $s \in \underline{A}_{X}(U)=A$, and $U(U, s)$ is identified with the usual basic open subset $D(s) \subseteq X$ under the bijections above. This proves that the LRS morphisms in question are isomorphisms on the level of spaces, so it remains only to prove that they are isomorphisms on the level of sheaves of rings, which we can check on stalks using the description of the stalks of a localization in Proposition 1.

Remark 4. If $X \in \mathbf{L R S}$, and $M$ is a prime system on $X$, the map $\pi: X^{l o c} \rightarrow X$ is not generally a morphism in LRS, even though $X, X^{l o c} \in$ LRS. For ex- 
ample, if $X$ is a point whose "sheaf" of rings is a local ring $(A, \mathfrak{m})$, and $M=\{\mathfrak{p}\}$ for some $\mathfrak{p} \neq \mathfrak{m}$, then $X^{\text {loc }}$ is a point with the "sheaf" of rings $A_{p}$, and the "stalk" of $\pi^{\#}$ is the localization map $l: A \rightarrow A_{p}$. Even though $A, A$ are local, this is not a local morphism because $l^{-1}\left(\mathfrak{p} A_{\mathfrak{p}}\right)=\mathfrak{p} \neq \mathfrak{m}$.

\section{Applications}

In this section we give some applications of localization of ringed spaces.

\subsection{Inverse Limits}

We first prove that LRS has all inverse limits.

Theorem 4. The category PRS has all inverse limits, and both the localization functor PRS $\rightarrow$ LRS and the forgetful functor PRS $\rightarrow$ RS preserve them.

Proof. Suppose $i \mapsto\left(X_{i}, M_{i}\right)$ is an inverse limit system in PRS . Let $X$ be the inverse limit of $i \mapsto X_{i}$ in Top and let $\pi_{i}: X \rightarrow X_{i}$ be the projection. Let $\mathcal{O}_{X}$ be the direct limit of $i \mapsto \pi_{i}^{-1} \mathcal{O}_{X_{i}}$ in $\operatorname{Rings}(X)$ and let $\pi_{i}^{\#}: \pi_{i}^{-1} \mathcal{O}_{X_{i}} \rightarrow \mathcal{O}_{X}$ be the structure map to the direct limit, so we may regard $X=\left(X, \mathcal{O}_{X}\right)$ as a ringed space and $\pi_{i}$ as a morphism of ringed spaces $X \rightarrow X_{i}$. It immediate from the definition of a morphism in $\mathbf{R S}$ that $X$ is the inverse limit of $i \mapsto X_{i}$ in RS. Let $M_{i}$ be the prime system on $X$ given by the inverse limit (intersection) of the $\pi_{i}^{*} M_{i}$. Then it is clear from the definition of a morphism in PRS that $(X, M)$ is the inverse limit of $i \mapsto\left(X_{i}, M_{i}\right)$, but we will spell out the details for the sake of concreteness and future use.

Given a point $x=\left(x_{i}\right) \in X$, we have defined $M_{x}$ to be the set of $z \in \operatorname{Spec}_{X, x}$ such that

$\pi_{i, x}^{-1}(z) \in M_{x_{i}} \subseteq \operatorname{Spec} \mathcal{O}_{X_{i}, x_{i}}$ for every $i$, so that $\pi_{i}$ defines a PRS morphism $\pi_{i}:(X, M) \rightarrow\left(X_{i}, M_{i}\right)$. To see that $(X, M)$ is the direct limit of $i \mapsto\left(X_{i}, M_{i}\right)$ suppose $f_{i}:(Y, N) \rightarrow\left(X_{i}, M_{i}\right)$ are morphisms defining a natural transformation from the constant functor $i \mapsto(Y, N)$ to $i \mapsto\left(X_{i}, M_{i}\right)$. We want to show that there is a unique PRS morphism $f:(Y, N) \rightarrow(X, M)$ with $\pi_{i} f=f_{i}$ for all $i$. Since $X$ is the inverse limit of $i \mapsto X$ in RS, we know that there is a unique map of ringed spaces $f: Y \rightarrow X$ with $\pi_{i} f=f_{i}$ for all $i$, so it suffices to show that this $f$ is a PRS morphism. Let $y \in Y, \quad z \in N_{y}$. We must show $f_{y}^{-1}(z) \in M_{f(x)}$. By definition of $M$, we must show

$\left(\pi_{i}\right)_{f(x)}^{-1}\left(f_{y}^{-1}(z)\right) \in M_{\pi_{i}(f(x))}=M_{f_{i}(y)}$ for every $i$. But

$\pi_{i} f=f_{i}$ implies $f_{y}\left(\pi_{i}\right)_{f(x)}=\left(f_{i}\right)_{y}$, so

$\left(\pi_{i}\right)_{f(x)}^{-1}\left(f_{y}^{-1}(z)\right)=\left(f_{i}\right)_{y}^{-1}(z)$ is in $M_{f_{i}(y)}$ because $f_{i}$ is a PRS morphism.

The fact that the localization functor preserves inverse limits follows formally from the adjointness in Theorem 2.

Corollary 5. The category LRS has all inverse limits.

Proof. Suppose $i \mapsto X_{i}$ is an inverse limit system in LRS . Composing with $\mathcal{M}$ yields an inverse limit system $i \mapsto\left(X_{i}, \mathcal{M}_{X_{i}}\right)_{\text {loc }}$ in PRS. By the theorem, the localization $(X, M)^{\text {loc }}$ of the inverse limit $(X, M) \underset{\text { of }}{\text { of }}$ $i \mapsto\left(X_{i}, \mathcal{M}_{X_{i}}\right)$ is the inverse limit of $i \mapsto\left(X_{i}, \mathcal{M}_{X_{i}}\right)^{\text {Ioc }}$ in LRS. But localization retracts $\mathcal{M}$ (Theorem 2) so $i \mapsto\left(X_{i}, \mathcal{M}_{X_{i}}\right)^{\text {Ioc }}$ is our original inverse limit system $i \mapsto X_{i}$.

We can also obtain the following result of $\mathrm{C}$. Chevalley mentioned in [Hak IV.2.4].

Corollary 6 The functor

$$
\begin{aligned}
\mathbf{R S} & \rightarrow \text { LRS } \\
X & \mapsto\left(X, \mathcal{T}_{X}\right)^{l o c}
\end{aligned}
$$

is right adjoint to the inclusion $\mathbf{L R S} \rightarrow \mathbf{R S}$.

Proof. This is immediate from the adjointness property of localization in Theorem 2 and the adjointness property of the functor $\mathcal{T}$ : For $Y \in \mathbf{L R S}$ we have

$$
\begin{aligned}
& \operatorname{Hom}_{\mathrm{LRS}}\left(Y,\left(X, \mathcal{T}_{X}\right)^{\text {loc }}\right) \\
& =\operatorname{Hom}_{\mathrm{PRS}}\left(\left(Y, \mathcal{M}_{Y}\right),\left(X, \mathcal{T}_{X}\right)\right) \\
& =\operatorname{Hom}_{\mathrm{RS}}(Y, X) .
\end{aligned}
$$

Our next task is to compare inverse limits in Sch to those in LRS. Let $* \in$ Top be "the" punctual space (terminal object), so $\operatorname{Rings}(*)=$ Rings. The functor

$$
\begin{aligned}
\text { Rings } & \rightarrow \mathbf{R S} \\
A & \mapsto\left({ }^{*}, A\right)
\end{aligned}
$$

is clearly left adjoint to

$$
\begin{aligned}
\Gamma: \mathbf{R S}^{\mathrm{op}} & \rightarrow \text { Rings } \\
X & \mapsto \Gamma\left(X, \mathcal{O}_{X}\right) .
\end{aligned}
$$

By Lemma 3 (or Proposition 1) we have

$$
\begin{aligned}
\mathcal{T}(*, A)^{l o c} & :=(*, A, \operatorname{Spec} A)^{l o c} \\
& =\operatorname{Spec} A .
\end{aligned}
$$

Theorem 2 yields an easy proof of the following result, which can be found in the Errata for [EGA I.1.8] printed at the end of [EGA II].

Proposition 7. For $A \in$ Rings , $X \in \mathbf{L R S}$, the natural map

$$
\operatorname{Hom}_{\text {LRS }}(X, \operatorname{Spec} A) \rightarrow \operatorname{Hom}_{\text {Rings }}\left(A, \Gamma\left(X, \mathcal{O}_{X}\right)\right)
$$

is bijective, so Spec:Rings $\rightarrow$ LRS is left adjoint to $\Gamma:$ LRS $^{\text {op }} \rightarrow$ Rings .

Proof. This is a completely formal consequence of various adjunctions: 


$$
\begin{aligned}
\operatorname{Hom}_{\mathbf{L R S}}(X, \operatorname{Spec} A) & =\operatorname{Hom}_{\mathbf{L R S}}\left(X, \mathcal{T}(*, A)^{\text {loc }}\right) \\
& =\operatorname{Hom}_{\mathbf{P R S}}\left(\left(X, \mathcal{M}_{X}\right), \mathcal{T}(*, A)\right) \\
& =\operatorname{Hom}_{\mathbf{R S}}(X,(*, A)) \\
& =\operatorname{Hom}_{\text {Rings }}\left(A, \Gamma\left(X, \mathcal{O}_{X}\right)\right) .
\end{aligned}
$$

Theorem 8. The category Sch has all finite inverse limits, and the inclusion $\mathbf{S c h} \rightarrow \mathbf{L R S}$ preserves them.

Proof. It is equivalent to show that, for a finite inverse limit system $i \mapsto X_{i}$ in Sch, the inverse limit $X$ in LRS is a scheme. It suffices to treat the case of (finite) products and equalizers. For products, suppose $\left\{X_{i}\right\}$ is a finite set of schemes and $X=\prod_{i} X_{i}$ is their product in LRS. We want to show $X$ is a scheme. Let $\bar{x}$ be a point of $X$, and let $x=\left(x_{i}\right) \in \prod_{i}^{\mathrm{RS}} X_{i}$ be its image in the ringed space product. Let $U_{i}=\operatorname{Spec} A_{i}$ be an open affine neighborhood of $x_{i}$ in $X_{i}$. As we saw above, the map $X \rightarrow \prod_{i}^{\mathrm{RS}} X_{i}$ is a localization and, as mentioned in Remark 3, it follows that the product $U:=\prod_{i} U_{\bar{i}}$ of the $U_{i}$ in LRS is an open neighborhood of $X$ in $X,{ }^{7}$, so it remains only to prove that there is an isomorphism $U \cong \operatorname{Spec} \otimes_{i} A_{i}$, hence $U$ is affine. ${ }^{8}$ Indeed, we can see immediately from Proposition 7 that $U$ and $\operatorname{Spec} \otimes_{i} A_{i}$ represent the same functor on LRS :

$$
\begin{aligned}
\operatorname{Hom}_{\text {LRS }}(Y, U) & =\prod_{i} \operatorname{Hom}_{\text {LRS }}\left(Y, U_{i}\right) \\
& =\prod_{i} \operatorname{Hom}_{\text {Rings }}\left(A_{i}, \Gamma\left(Y, \mathcal{O}_{Y}\right)\right) \\
& =\operatorname{Hom}_{\text {Rings }}\left(\otimes_{i} A_{i}, \Gamma\left(Y, \mathcal{O}_{Y}\right)\right) \\
& =\operatorname{Hom}_{\text {LRS }}\left(Y, \operatorname{Spec} \otimes_{i} A_{i}\right) .
\end{aligned}
$$

The case of equalizers is similar: Suppose $X$ is the LRS equalizer of morphisms $f, g: Y \rightrightarrows Z$ of schemes, and $x \in X$. Let $y \in Y$ be the image of $x$ in $Y$, so $f(y)=g(y)=: z$. Since $Y, Z$ are schemes, we can find affine neighborhoods $V=\operatorname{Spec} B$ of $y$ in $Y$ and $W=\operatorname{Spec} A$ of $z$ in $Z$ so that $f, g$ take $V$ into $W$. As before, it is clear that the equalizer $U$ of $f|V, g| V: V \rightrightarrows W$ in LRS is an open neighborhood of $x \in X$, and we prove exactly as above that $U$ is

${ }^{7}$ This is the only place we need "finite". If $\left\{X_{i}\right\}$ were infinite, the topological space product of the $U_{i}$ might not be open in the topology on the topological space product of the $X_{i}$ because the product topology only allows "restriction in finitely many coordinates".

${ }^{8}$ There would not be a problem here even if $\left\{X_{i}\right\}$ were infinite: Rings has all direct and inverse limits, so the (possibly infinite) tensor product $\otimes_{i} A_{i}$ over $\mathbb{Z}$ (coproduct in Rings) makes sense. Our proof therefore shows that any inverse limit (not necessarily finite) of affine schemes, taken in LRS, is a scheme. affine by showing that it is isomorphic to Spec of the coequalizer

$$
C=B /\left\langle\left\{f^{\#}(a)-g^{\#}(a): a \in A\right\}\right\rangle
$$

of $f^{\#}, g^{\#}: A \rightrightarrows B$ in Rings .

Remark 5. The basic results concerning the existence of inverse limits in LRS and their coincidence with inverse limits in Sch are, at least to some extent, "folk theorems". I do not claim originality here. The construction of fibered products in LRS can perhaps be attributed to Hanno Becker [HB], and the fact that a cartesian diagram in Sch is also cartesian in LRS is implicit in the [EGA] Erratum mentioned above.

Remark 6. It is unclear to me whether the 2-category of locally ringed topoi has 2-fibered products, though Hakim seems to want such a fibered product in [Hak V.3.2.3].

\subsection{Fibered Products}

In this section, we will more closely examine the construction of fibered products in LRS and explain the relationship between fibered products in LRS and those in RS. By Theorem 8, the inclusion Sch $\longmapsto$ LRS preserves inverse limits, so these results will generalize the basic results comparing fibered products in Sch to those in RS (the proofs will become much more transparent as well).

Definition 5. Suppose

$(A, \mathfrak{m}, k),\left(B_{1}, \mathfrak{m}_{1}, k_{1}\right),\left(B_{2}, \mathfrak{m}_{2}, k_{2}\right) \in \mathbf{L A n}$ and $f_{i}: A \rightarrow B_{i}$ are LAn morphisms, so $f_{i}^{-1}\left(\mathfrak{m}_{i}\right)=\mathfrak{m}$ for $i=1,2$. Let $i_{j}: B_{j} \rightarrow B_{1} \otimes_{A} B_{2} \quad(j=1,2)$ be the natural maps. Set

$$
\begin{aligned}
S\left(A, B_{1}, B_{2}\right) & :=\left\{\mathfrak{p} \in \operatorname{Spec}\left(B_{1} \otimes_{A} B_{2}\right): i_{1}^{-1}(\mathfrak{p})\right. \\
& \left.=\mathfrak{m}_{1}, i_{2}^{-1}(\mathfrak{p})=\mathfrak{m}_{2}\right\} .
\end{aligned}
$$

Note that the kernel $K$ of the natural surjection

$$
\begin{aligned}
B_{1} \otimes_{A} B_{2} & \rightarrow k_{1} \otimes_{k} k_{2} \\
b_{1} \otimes b_{2} & \mapsto\left[b_{1}\right] \otimes\left[b_{2}\right]
\end{aligned}
$$

is generated by the expressions $m_{1} \otimes 1$ and $1 \otimes m_{2}$, where $m_{i} \in \mathfrak{m}_{i}$, so

$$
\operatorname{Spec}\left(k_{1} \otimes_{k} k_{2}\right) \longmapsto \operatorname{Spec}\left(B_{1} \otimes_{A} B_{2}\right)
$$

is an isomorphism onto $S\left(A, B_{1}, B_{2}\right)$. In particular,

$$
S\left(A, B_{1}, B_{2}\right)=\left\{\mathfrak{p} \in \operatorname{Spec}\left(B_{1} \otimes_{A} B_{2}\right): K \subseteq \mathfrak{p}\right\}
$$

is closed in $\operatorname{Spec}\left(B_{1} \otimes_{A} B_{2}\right)$.

The subset $S\left(A, B_{1}, B_{2}\right)$ enjoys the following important property: Suppose $g_{i}:\left(B_{i}, \mathfrak{m}_{i}\right) \rightarrow(C, \mathfrak{n}), i=1,2$, are LAn morphisms with $g_{1} f_{1}=g_{2} f_{2}$ and 
$h=\left(f_{1}, f_{2}\right): B_{1} \otimes_{A} B_{2} \rightarrow C$ is the induced map. Then $h^{-1}(\mathfrak{n}) \in S\left(A, B_{1}, B_{2}\right)$. Conversely, every

$\mathfrak{p} \in S\left(A, B_{1}, B_{2}\right)$ arises in this manner: take

$C=\left(B_{1} \otimes_{A} B_{2}\right)_{\mathfrak{p}}$.

Setup: We will work with the following setup throughout this section. Let $f_{1}: X_{1} \rightarrow Y, f_{2}: X_{2} \rightarrow Y$ be morphisms in LRS. From the universal property of fiber products we get a natural "comparison" map

$$
\eta: X_{1} \times_{Y}^{\mathbf{L R S}} X_{2} \rightarrow X_{1} \times_{Y}^{\mathrm{RS}} X_{2} .
$$

Let $\pi_{i}: X_{1} \times_{Y}^{\mathrm{RS}} X_{2} \rightarrow X_{i} \quad(i=1,2)$ denote the projections and let $g:=f_{1} \pi_{1}=f_{2} \pi_{2}$. Recall that the structure sheaf of $X_{1} \times \times_{Y}^{\mathrm{RS}} X_{2}$ is $\pi_{1}^{-1} \mathcal{O}_{X_{1}} \otimes_{q^{-1} \mathcal{O}_{Y}} \pi_{2}^{-1} \mathcal{O}_{X_{2}}$. In particular, the stalk of this structure ${ }^{-1}$ sheaf at a point $x=\left(x_{1}, x_{2}\right) \in X_{1} \times_{Y}^{\mathbf{R S}} X_{2}$ is $\mathcal{O}_{X_{1}, x_{1}} \otimes_{\mathcal{O}_{Y, y}} \mathcal{O}_{X_{2}, x_{2}}$, where

$$
y:=g(x)=f_{1}\left(x_{1}\right)=f_{2}\left(x_{2}\right) \text {. }
$$

In this situation, we set

$$
S\left(x_{1}, x_{2}\right):=S\left(\mathcal{O}_{Y, y}, \mathcal{O}_{X_{1}, x_{1}}, \mathcal{O}_{X_{2}, x_{2}}\right)
$$

to save notation.

Theorem 9. The comparison map $\eta$ is surjective on topological spaces. More precisely, for any

$x=\left(x_{1}, x_{2}\right) \in X_{1} \times_{Y}^{\mathbf{R S}} X_{2}, \eta^{-1}(x)$ is in bijective correspondence with the set $S\left(x_{1}, x_{2}\right)$, and in fact, there is an LRS isomorphism

$$
\begin{aligned}
\eta^{-1}(x) & :=X_{1} \times_{Y}^{\mathbf{L R S}} X_{2} \times_{X_{1} \times \mathbf{R S}_{Y}}\left(x, \mathcal{O}_{X_{1}, x_{1}} \otimes_{\mathcal{O}_{Y, y}} \mathcal{O}_{X_{2}, x_{2}}\right) \\
& =\operatorname{Spec}_{{ }_{X_{1}, x_{1}} \otimes_{\mathcal{O}_{Y, y}} \mathcal{O}_{X_{2}, x_{2}}} S\left(x_{1}, x_{2}\right) .
\end{aligned}
$$

In particular, $\eta^{-1}(x)$ is isomorphic as a topological space to $\operatorname{Speck}\left(x_{1}\right) \otimes_{k(y)} k\left(x_{2}\right)$ (but not as a ringed space). The stalk of $\eta$ at $z \in S\left(x_{1}, x_{2}\right)$ is identified with the localization map

$$
\mathcal{O}_{X, x_{1}} \otimes_{\mathcal{O}_{Y, y}} \mathcal{O}_{X, x_{2}} \rightarrow\left(\mathcal{O}_{X, x_{1}} \otimes_{\mathcal{O}_{Y, y}} \mathcal{O}_{X, x_{2}}\right)_{z}
$$

In particular, $\eta$ is a localization morphism (Definition 1).

Proof. We saw in $\S 3.1$ that the comparison map $\eta$ is identified with the localization of $X_{1} \times_{Y}^{\mathbf{R S}} X_{2}$ at the prime system $\left(x_{1}, x_{2}\right) \mapsto S\left(x_{1}, x_{2}\right)$, so these results follow from Proposition 1.

Remark 7. When $X_{1}, X_{2}, Y \in$ Sch, the first statement of Theorem 9 is [EGA I.3.4.7].

Remark 8. The fact that $\eta$ is a localization morphism is often implicitly used in the theory of the cotangent complex.

Definition 6. Let $f: X \rightarrow Y$ be an LRS morphism. A point $x \in X$ is called rational over $Y$ (or "over $y:=f(x)$ " or "with respect to $f$ ") iff the map on residue fields $\bar{f}_{x}: k(y) \rightarrow k(x)$ is an isomorphism (equivalently: is surjective).
Corollary 10. Suppose $x_{1} \in X_{1}$ is rational over $Y$ (i.e. with respect to $\left.f_{1}: X_{1} \rightarrow Y\right)$ ). Then for any $x=\left(x_{1}, x_{2}\right) \in X_{1} \times_{Y}^{\mathbf{R S}} X_{2}$, the fiber $\eta^{-1}(x)$ of the comparison map $\eta$ is punctual. In particular, if every point of $X_{1}$ is rational over $Y$, then $\eta$ is bijective.

Proof. Suppose $x_{1} \in X_{1}$ is rational over $Y$. Suppose $x=\left(x_{1}, x_{2}\right) \in X_{1} \times{ }^{\mathrm{RS}} X_{2}$. Set $y:=f_{1}\left(x_{1}\right)=f_{2}\left(x_{2}\right)$. Since $x_{1}$ is rational, $k(y) \cong k\left(x_{1}\right)$, so

Spec $k\left(x_{1}\right) \otimes_{k(y)} k\left(x_{2}\right) \cong \operatorname{Spec} k\left(x_{2}\right)$ has a single element. On the other hand, we saw in Definition 5 that this set is in bijective correspondence with the set

$$
S\left(x_{1}, x_{2}\right) \subseteq \operatorname{Spec}\left(\mathcal{O}_{X_{1}, x_{1}} \otimes_{\mathcal{O}_{Y, y}} \mathcal{O}_{X_{2}, x_{2}}\right)
$$

appearing in Theorem 9, so that same theorem says that $\eta^{-1}(x)$ consists of a single point.

Remark 9. Even if every $x_{1} \in X$ is rational over $Y$, the comparison map

$$
\eta: X_{1} \times_{Y}^{\mathbf{L R S}} X_{2} \rightarrow X_{1} \times_{Y}^{\mathbf{R S}} X_{2}
$$

is not generally an isomorphism on topological spaces, even though it is bijective. The topology on $X_{1} \times{ }_{Y}^{\text {LRS }} X_{2}$ is generally much finer than the product topology. In this situation, the set $S\left(x_{1}, x_{2}\right)$ always consists of a single element $z\left(x_{1}, x_{2}\right)$ : namely, the maximal ideal of $\mathcal{O}_{X_{1}, x_{1}} \otimes_{\mathcal{O}_{Y, y}} \mathcal{O}_{X_{2}, x_{2}}$ given by the kernel of the natural

$$
\mathcal{O}_{X_{1}, x_{1}} \otimes_{\mathcal{O}_{Y, y}} \mathcal{O}_{X_{2}, x_{2}} \rightarrow k\left(x_{1}\right) \otimes_{k(y)} k\left(x_{2}\right)=k\left(x_{2}\right) .
$$

If we identify $X_{1} \times_{Y}^{\mathrm{LRS}} X_{2}$ and $X_{1} \times_{Y}^{\mathrm{RS}} X_{2}$ as sets via $\eta$, then the "finer" topology has basic open sets

$$
U\left(U_{1} \times_{Y} U_{2}, s\right):=\left\{\left(x_{1}, x_{2}\right) \in U_{1} \times_{Y} U_{2}: s_{\left(x_{1}, x_{2}\right)} \notin Z\left(x_{1}, x_{2}\right)\right\}
$$

as $U_{1}, U_{2}$ range over open subsets of $X_{1}, X_{2}$ and $s$ ranges over

$$
\left(\pi_{1}^{-1} \mathcal{O}_{X_{1}} \otimes_{g^{-1} \mathcal{O}_{Y}} \pi_{2}^{-1} \mathcal{O}_{X_{2}}\right)\left(U_{1} \times_{Y} U_{2}\right) .
$$

This set is not generally open in the product topology because the stalks of

$$
\pi_{1}^{-1} \mathcal{O}_{X_{1}} \otimes{ }_{g^{-1} \mathcal{O}_{Y}} \pi_{2}^{-1} \mathcal{O}_{X_{2}}
$$

are not generally local rings, so not being in $z\left(x_{1}, x_{2}\right)$ does not imply invertibility, hence is not generally an open condition on $\left(x_{1}, x_{2}\right)$.

Remark 10. On the other hand, sometimes the topologies on $X_{1}, X_{2}$ are so fine that the sets $U\left(U_{1} \times_{Y} U_{2}, s\right)$ are easily seen to be open in the product topology. For example, suppose $k$ is a topological field. ${ }^{9}$ Then one often works in the full subcategory $C$ of locally ringed spaces over $k$ consisting of those

${ }^{9}$ I require all finite subsets of $k$ to be closed in the definition of "topological field". 
$X \in \mathbf{L R S} / k$ satisfying the conditions:

1) Every point $x \in X$ is a $k$ point: the composition $k \rightarrow \mathcal{O}_{X, x} \rightarrow k(x)$ yields an isomorphism $k=k(x)$ for every $x \in X$.

2) The structure sheaf $\mathcal{O}_{X}$ is continuous for the topology on $k$ in the sense that, for every $(U, s) \in \operatorname{Sec} \mathcal{O}_{X}$, the function

$$
\begin{gathered}
s\left(\_\right): U \rightarrow k \\
x \mapsto s(x)
\end{gathered}
$$

is a continuous function on $U$. Here $s(x) \in k(x)$ denotes the image of the stalk $s_{x} \in \mathcal{O}_{X, X}$ in the residue field $k(x)=\mathcal{O}_{X, x} / \mathfrak{m}_{x}$, and we make the identification $k=k(x)$ using 1$)$.

One can show that fiber products in $C$ are the same as those in LRS and that the forgetful functor $C \rightarrow$ Top preserves fibered products (even though $C \rightarrow \mathbf{R S}$ may not). Indeed, given

$s \in\left(\pi_{1}^{-1} \mathcal{O}_{X_{1}} \otimes_{g^{-1} \mathcal{O}_{Y}} \pi_{2}^{-1} \mathcal{O}_{X_{2}}\right)\left(U_{1} \times_{Y} U_{2}\right)$, the set

$U\left(U_{1} \times_{Y} U_{2}, s\right)$ is the preimage of $k^{*} \subseteq k$ under the map $s\left({ }_{-}\right)$, and we can see that $s\left({ }_{-}\right)$is continuous as follows: By viewing the sheaf theoretic tensor product as the sheafification of the presheaf tensor product we see that, for any point $\left(x_{1}, x_{2}\right) \in U_{1} x_{Y} U_{2}$, we can find a neighborhood $V_{1} \times_{Y} V_{2}$ of $\left(x_{1}, x_{2}\right)$ contained in $U_{1} \times_{Y} U_{2}$ and sections $a_{1}, \cdots, a_{n} \in \mathcal{O}_{X_{1}}\left(V_{1}\right)$,

$b_{1}, \cdots, b_{n} \in \mathcal{O}_{X_{2}}\left(V_{2}\right)$ such that the stalk $s_{X_{I^{\prime}}, x_{2^{\prime}}}$ agrees with $\sum_{i}\left(a_{i}\right)_{x_{1^{\prime}}} \otimes\left(b_{i}\right)_{x_{2^{\prime}}}$ at each $\left(x_{1^{\prime}}, x_{2^{\prime}}\right) \in V_{1} \times_{Y} V_{2}$. In particular, the function $s\left({ }_{-}\right)$agrees with the function

$$
\left(x_{1^{\prime}}, x_{2^{\prime}}\right) \mapsto \sum_{i} a_{i}\left(x_{1^{\prime}}\right) b_{i}\left(x_{2^{\prime}}\right) \in k
$$

on $V_{1} \times_{Y} V_{2}$. Since this latter function is continuous in the product topology on $V_{1} \times_{Y} V_{2}$ (because each $a_{i}\left(\_\right)$, $b_{i}\left(\_\right)$is continuous) and continuity is local, $s\left({ }_{-}\right)$is continuous.

Corollary 11. Suppose $\left(f_{1}\right)_{x_{1}}: \mathcal{O}_{Y, f\left(x_{1}\right)} \rightarrow \mathcal{O}_{X_{1}, x_{1}}$ is surjective for every $x_{1} \in X_{1}$. Then the comparison map $\eta$ is an isomorphism. In particular, $\eta$ is an isomorphism under either of the following hypotheses:

1) $f_{1}$ is an immersion.

2) $f_{1}: \operatorname{Spec} k(y) \rightarrow Y$ is the natural map associated to a point $y \in Y$.

Proof. It is equivalent to show that $X:=X_{1} \times_{Y}^{\mathrm{RS}} X_{2}$ is in LRS and the structure maps $\pi_{i}: X \rightarrow X_{i}$ are LRS morphisms. Say $x=\left(x_{1}, x_{2}\right) \in X$ and let $y:=f_{1}\left(x_{1}\right)=f_{2}\left(x_{2}\right)$. By construction of $X$, we have a pushout diagram of rings

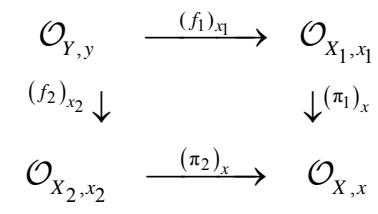

hence it is clear from surjectivity of $\left(f_{1}\right)_{x_{1}}$ and locality of $\left(f_{2}\right)_{x_{2}}$ that $\mathcal{O}_{X, x}$ is local and $\left(\pi_{1}\right)_{x},\left(\pi_{2}\right)_{x}$ are LAn morphisms.

Corollary 12. Suppose

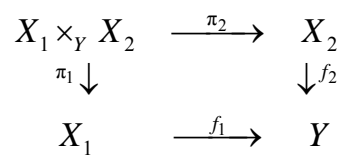

is a cartesian diagram in LRS. Then:

1) If $z \in X_{1} \times_{Y} X_{2}$ is rational over $Y$, then $\eta^{-1}(\eta(z))=\{z\}$.

2) Let $\left(x_{1}, x_{2}\right) \in X_{1} x_{Y}^{\mathrm{RS}} X_{2}$, and let

$y:=\pi_{1}\left(x_{1}\right)=\pi_{2}\left(x_{2}\right)$. Suppose $k\left(x_{2}\right)$ is isomorphic, as a field extesion of $k(y)$, to a subfield of $k\left(x_{1}\right)$. Then there is a point $z \in X_{1} \times_{Y}^{\text {sch }} X_{2}$ rational over $X_{1}$ with $\pi_{i}(z)=x_{i}, i=1,2$.

Proof. For 1), set $\left(x_{1}, x_{2}\right):=\eta(z)$, $y:=\pi_{1}\left(x_{1}\right)=\pi_{2}\left(x_{2}\right)$. Then we have a commutative diagram

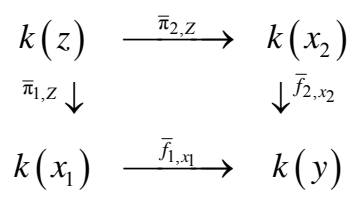

of residue fields. By hypothesis, the compositions $k(y) \rightarrow k\left(x_{i}\right) \rightarrow k(z)$ are isomorphisms for $i=1,2$, so it must be that every map in this diagram is an isomorphism, hence the diagram is a pushout. On the other hand, according to the first statement of Theorem 9, $\eta^{-1}(\eta(z))$ is in bijective correspondence with

$$
\operatorname{Spec}\left(k\left(x_{1}\right) \otimes_{k(y)} k\left(x_{2}\right)\right)=\operatorname{Speck}(z),
$$

which is punctual.

For 2), let $i: k\left(x_{2}\right) \longmapsto k\left(x_{1}\right)$ be the hypothesized morphism of field extensions of $k(y)$. By the universal property of the LRS fibered product $X_{1} \times_{Y} X_{2}$, the maps

$$
\begin{gathered}
\left(x_{2}, i\right): \operatorname{Speck}\left(x_{1}\right) \rightarrow X_{2} \\
x_{1}: \operatorname{Speck}\left(x_{1}\right) \rightarrow X_{1}
\end{gathered}
$$

give rise to a map

$$
g: \operatorname{Speck}\left(x_{1}\right) \rightarrow X_{1} \times_{Y} X_{2} .
$$

Let $z \in X_{1} \times_{Y} X_{2}$ be the point corresponding to this map. Then we have a commutative diagram of residue fields

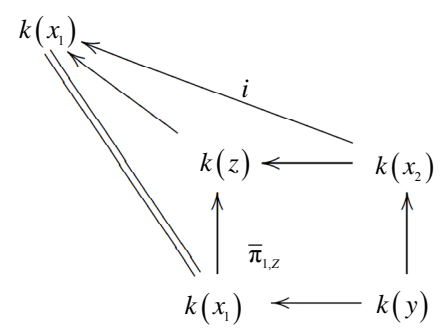


so $\bar{\pi}_{1, z}: k\left(x_{1}\right) \rightarrow k(z)$ must be an isomorphism.

\subsection{Spec Functor}

Suppose $\quad X \in \mathbf{L R S}$ and $f: \mathcal{O}_{X} \rightarrow A$ is an $\mathcal{O}_{X}$ algebra. Then $f$ may be viewed as a morphism of ringed spaces $f:(X, A) \rightarrow\left(X, \mathcal{O}_{X}\right)=X$. Give $X$ the local prime system $\mathcal{M}_{X}$ as usual and $(X, A)$ the inverse image prime system $\mathcal{M}$ (Remark 1 ), so $f$ may be viewed as a PRS morphism

$$
f:\left(X, A, f^{*} \mathcal{M}_{X}\right) \rightarrow\left(X, \mathcal{O}_{X}, \mathcal{M}_{X}\right)
$$

Explicitly:

$$
\left(f^{*} \mathcal{M}_{X}\right)_{X}=\left\{\mathfrak{p} \in A_{x}: f_{X}^{-1}(\mathfrak{p})=\mathfrak{m}_{x} \subseteq \mathcal{O}_{X, X}\right\} .
$$

By Theorem 2, there is a unique LRS morphism

$$
\bar{f}:\left(X, A, f^{*} \mathcal{M}_{X}\right)^{\text {loc }} \rightarrow\left(X, \mathcal{O}_{X}, \mathcal{M}_{X}\right)^{\text {loc }}=X
$$

lifting $f$ to the localizations. We call

$$
\operatorname{Spec}_{X} A:=\left(X, A, f^{*} \mathcal{M}_{X}\right)^{\text {loc }}
$$

the spectrum (relative to $X$ ) of $A . \operatorname{Spec}_{X}$ defines a functor

$$
\operatorname{Spec}_{X}:\left(\mathcal{O}_{X} / \operatorname{Rings}(X)\right)^{\mathrm{op}} \rightarrow \mathbf{L R S} / X .
$$

Note that $\operatorname{Spec}_{X} \mathcal{O}_{X}=\left(X, \mathcal{O}_{X}, \mathcal{M}_{X}\right)^{\text {loc }}=X$ by Theorem 2 .

Our functor $\operatorname{Spec}_{X}$ agrees with the usual one (c.f. [Har II.Ex.5.17]) on their common domain of definition:

Lemma 13. Let $f: X \rightarrow Y$ be an affine morphism of schemes. Then $\operatorname{Spec}_{X} f_{*} \mathcal{O}_{X}$ (as defined above) is naturally isomorphic to $X$ in $\mathbf{L R S} / Y$.

Proof. This is local on $Y$, so we can assume $Y=\operatorname{Spec} A$ is affine, and hence $X=\operatorname{Spec} B$ is also affine, and $f$ corresponds to a ring map $f^{\#}: A \rightarrow B$. Then

$$
f_{*} \mathcal{O}_{X}=B^{\sim}=\underline{B}_{Y} \otimes_{\underline{A}_{Y}} \mathcal{O}_{Y},
$$

as $\mathcal{O}_{Y}$ algebras, and the squares in the diagram

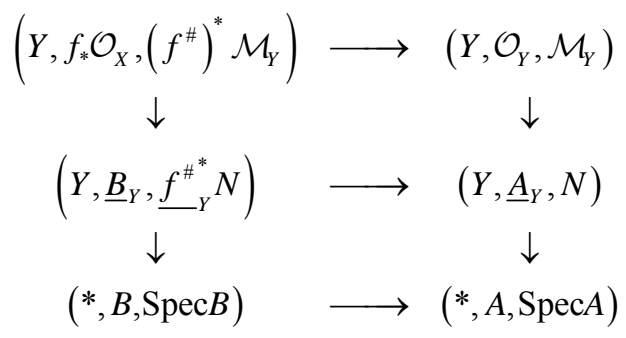

in PRS are cartesian in PRS, where $N$ is the prime system on $\left(Y, \underline{A}_{Y}\right)$ given by $N_{y}=\{y\}$ discussed in Lemma 3. According to that lemma, the right vertical arrows become isomorphisms upon localizing, and according to Theorem 4, the diagram stays cartesian upon localizing, so the left vertical arrows also become isomorphisms upon localizing, hence

$$
\begin{aligned}
\operatorname{Spec}_{Y} f_{*} \mathcal{O}_{X} & :=\left(Y, f_{*} \mathcal{O}_{X},\left(f^{\#}\right)^{*} \mathcal{M}_{Y}\right)^{l o c} \\
& =\operatorname{Spec} B \\
& =X .
\end{aligned}
$$

Remark 11. Hakim [Hak IV.1] defines a "Spec functor" from ringed topoi to locally ringed topoi, but it is not the same as ours on the common domain of definition. There is no meaningful situation in which Hakim's Spec functor agrees with the "usual" one. When $X$ "is" a locally ringed space, Hakim's $\operatorname{Spec} X$ "is" (up to replacing a locally ringed space with the corresponding locally ringed topos) our $\left(X, \mathcal{T}_{X}\right)^{\text {loc }}$. As mentioned in Remark 2, Hakim's theory of localization is only developed for the terminal prime system, which can be a bit awkward at times. For example, if $X$ is a locally ringed space at least one of whose local rings has positive Krull dimension, Hakim's sequence of spectra yields an infinite strictly descending sequence of RS morphisms

$$
\cdots \rightarrow \operatorname{Spec}(\operatorname{Spec} X) \rightarrow \operatorname{Spec} X \rightarrow X .
$$

The next results show that $\operatorname{Spec} X$ takes direct limits of $\mathcal{O}_{X}$ algebras to inverse limits in LRS and that Spec $X$ is compatible with changing the base $X$.

Lemma 14. The functor $\operatorname{Spec}_{X}$ preserves inverse limits.

Proof. Let $i \mapsto\left(f_{i}: \mathcal{O}_{X} \rightarrow A_{i}\right)$ be a direct limit system in $\mathcal{O}_{X} / \operatorname{Rings}(X)$, with direct limit $f: \mathcal{O}_{X} \rightarrow A$, and structure maps $j_{i}: A_{i} \rightarrow A$. We claim that

$\operatorname{Spec}_{X} A=\left(X, A, f^{*} \mathcal{M}_{X}\right)^{l o c}$ is the inverse limit of

$i \mapsto \operatorname{Spec}_{X} A_{i}=\left(X, A_{i}, f_{i}^{*} \mathcal{M}_{X}\right)^{\text {loc }}$. By Theorem 4, it is enough to show that $\left(X, A, f^{*} \mathcal{M}_{X}\right)$ is the inverse limit of $i \mapsto\left(X, A_{i}, f_{i}^{*} \mathcal{M}_{X}\right)$ in PRS. Certainly $(X, A)$ is the inverse limit of $i \mapsto\left(X, A_{i}\right)$ in RS, so we just need to show that $f^{*} \mathcal{M}_{X}=\cap_{i} j_{i}^{*}\left(f_{i}^{*} \mathcal{M}_{X}\right)$ as prime systems on $(X, A)$ (see the proof of Theorem 4), and this is clear because $j_{i} f_{i}=f$, so, in fact, $j_{i}^{*}\left(f_{i}^{*} \mathcal{M}_{X}\right)=f^{*} \mathcal{M}_{X}$ for every $i$.

Lemma 15. Let $f: X \rightarrow Y$ be a morphism of locally ringed spaces. Then for any $\mathcal{O}_{Y}$ algebra $g: \mathcal{O}_{Y} \rightarrow A$, the diagram

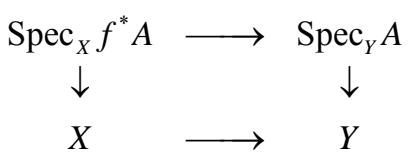

is cartesian in LRS.

Proof. Note $f^{*} A:=f^{-1} A \otimes_{f^{-1} \mathcal{O}_{Y}} \mathcal{O}_{X}$ as usual. One 


$$
\begin{array}{ccc}
\left(X, f^{*} A,\left(f^{-1} g\right)^{*} \mathcal{M}_{X}\right) & \longrightarrow\left(Y, A, g^{*} \mathcal{M}_{Y}\right) \\
\downarrow & \downarrow \\
\left(X, \mathcal{O}_{X}, \mathcal{M}_{X}\right) & \longrightarrow\left(Y, \mathcal{O}_{Y}, \mathcal{M}_{Y}\right)
\end{array}
$$

is cartesian in PRS so the result follows from Theorem 4.

Example 1. When $X$ is a scheme, but $A$ is not a coherent $\mathcal{O}_{X}$ module, $\operatorname{Spec}_{X} A$ may not be a scheme. For example, let $B$ be a local ring, $X:=\operatorname{Spec} B$, and let $x$ be the unique closed point of $X$. Let $A:=x_{*} B \in \operatorname{Rings}(X)$ be the skyscraper sheaf $B$ supported at $x$. Note $\mathcal{O}_{X, x}=B$ and

$$
\operatorname{Hom}_{\text {Rings }(X)}\left(\mathcal{O}_{X}, X_{*} B\right)=\operatorname{Hom}_{\text {Rings }}\left(\mathcal{O}_{X, X}, B\right),
$$

so we have a natural map $\mathcal{O}_{X} \rightarrow A$ in $\operatorname{Rings}(X)$ whose stalk at $X$ is $I d: B \rightarrow B$. Then

$\operatorname{Spec}_{X} A=(\{x\}, A)$ is the punctual space with "sheaf" of rings $A$, mapping in LRS to $X$ in the obvious manner. But $(\{x\}, A)$ is not a scheme unless $A$ is zero dimensional.

Here is another related pathology example: Proceed as above, assuming $B$ is a local domain which is not a field and let $K$ be its fraction field. Let $A:=x_{*} K$, and let $\mathcal{O}_{X} \rightarrow A$ be the unique map whose stalk at $x$ is $B \rightarrow K$. Then $\operatorname{Spec}_{X} A$ is empty.

Suppose $X$ is a scheme, and $A$ is an $\mathcal{O}_{X}$ algebra such that $\operatorname{Spec}_{X} A$ is a scheme. I do not know whether this implies that the structure morphism $\operatorname{Spec}_{X} A \rightarrow X$ is an affine morphism of schemes.

\subsection{Relative Schemes}

We begin by recalling some definitions.

Definition 7. ([SGA1], [Vis 3.1]) Let $F: C \rightarrow D$ be a functor. A $C$ morphism $f: c \rightarrow c^{\prime}$ is called cartesian (relative to $F$ ) iff, for any $C$ morphism $g: c^{\prime \prime} \rightarrow c^{\prime}$ and any $D$ morphism $h: F c^{\prime} \rightarrow F c^{\prime \prime}$ with $F g \circ h=F f$ there is a unique $C$ morphism $\bar{h}: c \rightarrow c^{\prime \prime}$ with $F \bar{h}=h$ and $f=g \bar{h}$. The functor $F$ is called a fibered category iff, for any $D$ morphism $f: d \rightarrow d^{\prime}$ and any object $c^{\prime}$ of $C$ with $F c^{\prime}=d^{\prime}$, there is a cartesian morphism $\bar{f}: c \rightarrow c^{\prime}$ with $F \bar{f}=f$. A morphism of fibered categories

$$
(F: C \rightarrow D) \rightarrow\left(F^{\prime}: C^{\prime} \rightarrow D^{\prime}\right)
$$

is a functor $G: C \rightarrow C^{\prime}$ satisfying $F^{\prime} G=F$ and taking cartesian arrows to cartesian arrows. If $D$ has a topology (i.e. is a site), then a fibered category $F: C \rightarrow D$ is called a stack iff, for any object $d \in D$ and any cover $\left\{d_{i} \rightarrow d\right\}$ of $d$ in $D$, the category $F^{-1}(d)$ is equivalent to the category $F\left(\left\{d_{i} \rightarrow d\right\}\right)$ of descent data (see [Vis 4.1]).
Every fibered category $F$ admits a morphism of fibered categories, called the associated stack, to a stack universal among such morphisms [Gir I.4.1.2].

Definition 8. ([Hak V.1]) Let $X$ be a ringed space. Define a category $\mathbf{S c h}_{X}^{\text {pre }}$ as follows. Objects of $\mathbf{S c h}_{X}^{\text {pre }}$ are pairs $\left(U, X_{U}\right)$ consisting of an open subset $U \subseteq X$ and a scheme $X_{U}$ over $\operatorname{Spec}_{X}(U)$. A morphism $\left(U, X_{U}\right) \rightarrow\left(V, X_{V}\right)$ is a pair $\left(U \rightarrow V, X_{U} \rightarrow X_{V}\right)$ consisting of an $\operatorname{Ouv}(X)$ morphism $U \rightarrow V$ (i.e. $U \subseteq V)$ and a morphism of schemes $X_{U} \rightarrow X_{V}$ making the diagram

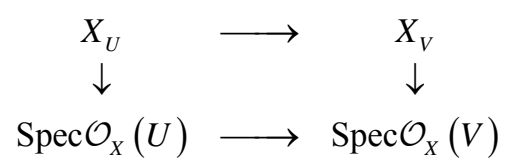

commute in Sch . The forgetful functor $\operatorname{Sch}_{X}^{\text {pre }} \rightarrow \operatorname{Ouv}(X)$ is clearly a fibered category, where a cartesian arrow is a $\mathbf{S c h}_{X}^{\text {pre }}$ morphism $\left(U \rightarrow V, X_{U} \rightarrow X_{V}\right)$ making (6) cartesian in Sch (equivalently in LRS ). Since $\operatorname{Ouv}(X)$ has a topology, we can form the associated stack $\mathbf{S c h}_{X}$. The category of relative schemes over $X$ is, by definition, the fiber category $\operatorname{Sch}_{X}(X)$ of $\operatorname{Sch}_{X}$ over the terminal object $X$ of $\operatorname{Ouv}(X)$.

(The definition of relative scheme makes sense for a ringed topos $X$ with trivial modifications.)

\subsection{Geometric Realization}

Now let $X$ be a locally ringed space. Following [Hak V.3], we now define a functor

$$
F_{X}: \operatorname{Sch}_{X}(X) \rightarrow \mathbf{L R S} / X
$$

called the geometric realization. Although a bit abstract, the fastest way to proceed is as follows:

Definition 9. Let $\mathbf{L R S}_{X}$ be the category whose objects are pairs $\left(U, X_{U}\right)$ consisting of an open subset $U \subseteq X$ and a locally ringed space $X_{U}$ over $\left(U, \mathcal{O}_{X} \mid U\right)$, and where a morphism $\left(U, X_{U}\right) \rightarrow\left(V, X_{V}\right)$ is a pair $\left(U \rightarrow V, X_{U} \rightarrow X_{V}\right)$ consisting of an $\operatorname{Ouv}(X)$ morphism $U \rightarrow V$ (i.e. $U \subseteq V)$ and an LRS morphism $X_{U} \rightarrow X_{V}$ making the diagram

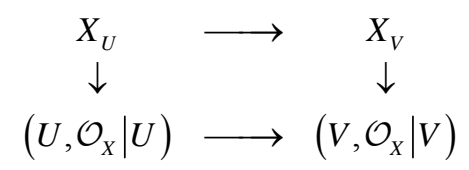

commute in LRS. The forgetful functor $\left(U, X_{U}\right) \mapsto U$ makes $\mathbf{L R S}_{X}$ a fibered category over $\operatorname{Ouv}(X)$ where a cartesian arrow is a morphism $\left(U \rightarrow V, X_{U} \rightarrow X_{V}\right)$ making (8) cartesian in LRS .

In fact the fibered category $\operatorname{LRS}_{X} \rightarrow \operatorname{Ouv}(X)$ is a stack: one can define locally ringed spaces and mor- 
phisms thereof over open subsets of $X$ locally. Using the universal property of stackification, we define $F_{X}$ to be the morphism of stacks (really, the corresponding morphism on fiber categories over the terminal object $X \in \operatorname{Ouv}(X))$ associated to the morphism of fibered categories

$$
\begin{gathered}
F_{X}^{\text {pre }}: \mathbf{S c h}_{X}^{\text {pre }} \rightarrow \mathbf{L R S}_{X} \\
\left(U, X_{U}\right) \mapsto\left(U, X_{U} \times_{\mathrm{SpecO}_{X}(U)}^{\mathrm{LRS}}\left(U, \mathcal{O}_{X} \mid U\right)\right) .
\end{gathered}
$$

The map $\left(U, \mathcal{O}_{X} \mid U\right) \rightarrow \operatorname{Spec} \mathcal{O}_{X}(U)$ is the adjunction morphism for the adjoint functors of Proposition 7. This functor clearly takes cartesian arrows to cartesian arrows.

Remark 12. Although we loosely follow [Hak V.3.2] in our construction of the geometric realization, our geometric realization functor differs from Hakim's on their common domain of definition.

\subsection{Relatively Affine Morphisms}

Let $f: X \rightarrow Y$ be an LRS morphism. Consider the following conditions:

RA1. Locally on $Y$ there is an $\mathcal{O}_{Y}(Y)$ algebra $A$ and a cartesian diagram

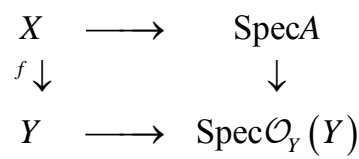

in LRS .

RA2. There is an $\mathcal{O}_{Y}$ algebra $A$ so that $f$ is isomorphic to $\operatorname{Spec}_{Y} A$ in $\mathbf{L R S} / Y$.

RA3. Same condition as above, but $A$ is required to be quasi-coherent.

RA4. For any $g: Z \rightarrow Y$ in LRS/ $Y$, the map

$$
\begin{aligned}
\operatorname{Hom}_{\mathbf{L R S} / Y}(Z, X) & \rightarrow \operatorname{Hom}_{\mathcal{O}_{Y} / \operatorname{Rings}(Y)}\left(f_{*} \mathcal{O}_{X}, g_{*} \mathcal{O}_{Z}\right) \\
h & \mapsto g_{*} h^{\#}
\end{aligned}
$$

is bijective.

Remark 13. The condition (RA1) is equivalent to both of the following conditions: [label $=$ RA1.., ref $=$ RA1]

RA1.1 Locally on $Y$ there is a ring homomorphism $A \rightarrow B$ and a cartesian diagram

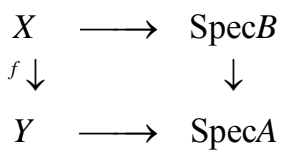

\section{in LRS .}

RA1.2. Locally on $Y$ there is an affine morphism of schemes $X^{\prime} \rightarrow Y^{\prime}$ and a cartesian diagram

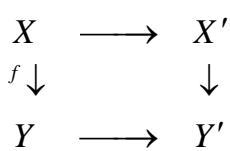

in LRS .

The above two conditions are equivalent by definition of an affine morphism of schemes, and one sees the equivalence of (RA1) and (RA1.1) using Proposition 7, which ensures that the map $Y \rightarrow \operatorname{Spec} A$ in (RA1) factors through $Y \rightarrow \operatorname{Spec}_{Y}(Y)$, hence

$$
\begin{aligned}
X & =Y \times_{\operatorname{Spec} A} \operatorname{Spec} B \\
& =Y \times_{\operatorname{Spec} O_{Y}(Y)} \operatorname{Spec} \mathcal{O}_{Y}(Y) \times_{\operatorname{Spec} A} \operatorname{Spec} B \\
& =Y \times_{\operatorname{Spec}_{Y}(Y)} \operatorname{Spec}\left(\mathcal{O}_{Y}(Y) \otimes_{A} B\right) .
\end{aligned}
$$

Each of these conditions has some claim to be the definition of a relatively affine morphism in LRS. With the exception of (2), all of the conditions are equivalent, when $Y$ is a scheme, to $f$ being an affine morphism of schemes in the usual sense. With the exception of (4), each condition is closed under base change. For each possible definition of a relatively affine morphism in LRS, one has a corresponding definition of relatively schematic morphism, namely: $f: X \rightarrow Y$ in LRS is relatively schematic iff, locally on $X, f$ is relatively affine.

The notion of "relatively schematic morphism" obtained from (1) is equivalent to: $f: X \rightarrow Y$ is in the essential image of the geometric realization functor $F_{Y}$.

\subsection{Monoidal Spaces}

The setup of localization of ringed spaces works equally well in other settings; for example in the category of monoidal spaces. We will sketch the relevant definitions and results. For our purposes, a monoid is a set $P$ equipped with a commutative, associative binary operation + such that there is an element $0 \in P$ with $0+p=p$ for all $p \in P$. A morphism of monoids is a map of sets that respects + and takes 0 to 0 . An ideal of a monoid $P$ is a subset $I \subseteq P$ such that $I+P \subseteq I$. An ideal $I$ is prime iff its complement is a submonoid (in particular, its complement must be non-empty). A submonoid whose complement is an ideal, necessarily prime, is called a face. For example, the faces of $\mathbb{N}^{2}$ are $\{(0,0)\}, \mathbb{N} \oplus 0$, and $0 \oplus \mathbb{N}$; the diagonal $\Delta: \mathbb{N} \mapsto \mathbb{N}^{2}$ is a submonoid, but not a face.

If $S \subseteq P$ is a submonoid, the localization of $P$ at $S$ is the monoid $S^{-1} P$ whose elements are equivalence classes $[p, s], \quad p \in P, \quad s \in S$ where $[p, s]=\left[p^{\prime}, s^{\prime}\right]$ iff there is some $t \in S$ with $t+p+s^{\prime}=t+p^{\prime}+s$, and where $[p, s]+\left[p^{\prime}, s^{\prime}\right]:=\left[p+p^{\prime}, s+s^{\prime}\right]$. The natural map $P \rightarrow S^{-1} P$ given by $p \mapsto[p, 0]$ is initial among mon- 
oid homomorphisms $h: P \rightarrow Q$ with $h(S) \subseteq Q^{*}$. The localization of a monoid at a prime ideal is, by definition, the localization at the complementary face.

A monoidal space $\left(X, \mathcal{M}_{X}\right)$ is a topological space $X$ equipped with a sheaf of monoids $\mathcal{M}_{X}$. Monoidal spaces form a category $M S$ where a morphism $f=\left(f, f^{\dagger}\right):\left(X, \mathcal{M}_{X}\right) \rightarrow\left(Y, \mathcal{M}_{Y}\right)$ consists of a continuous map $f: X \rightarrow Y$ together with a map $\mathcal{M}$ of sheaves of monoids on $X$. A monoidal space $\left(X, \mathcal{M}_{X}\right)$ is called local iff each stalk monoid $\mathcal{M}_{X}$ has a unique maximal ideal $\mathfrak{m}_{X}$. Local monoidal spaces form a category LMS where a morphism is a map of the underlying monoidal spaces such that each stalk map $f_{x}^{\dagger}: \mathcal{M}_{Y, f(x)} \rightarrow \mathcal{M}_{X, x}$ is local in the sense $\left(f^{\dagger}\right)^{-1} \mathfrak{m}_{f(x)}=\mathfrak{m}_{x}$. A primed monoidal space is a monoidal space equipped with a set of primes $M_{x}$ in each stalk monoid $\mathcal{M}_{X, x}$. The localization of a primed monoidal space is a map of monoidal spaces

$\left(X, \mathcal{M}_{X}, M\right)^{\text {loc }} \rightarrow\left(X, \mathcal{M}_{X}\right)$ from a local monoidal space constructed in an obvious manner analogous to the construction of $\S 2.2$ and enjoying a similar universal property. In particular, we let $\operatorname{Spec} P$ denote the localization of the punctual space with "sheaf" of monoids $P$ at the terminal prime system. A scheme over $\mathbb{F}_{1}$ is a locally monoidal space locally isomorphic to $\mathrm{Spec} P$ for various monoids $P$. (This is not my terminology.)

The same "general nonsense" arguments of this paper allow us to construct inverse limits of local monoidal spaces, to prove that a finite inverse limit of schemes over $\mathbb{F}_{1}$, taken in local monoidal spaces, is again a scheme over $\mathbb{F}_{1}$, to construct a relative Spec functor

$$
\operatorname{Spec}:\left(\mathcal{M}_{X} / \operatorname{Mon}(X)\right)^{\text {op }} \rightarrow \mathbf{L M S} /\left(X, \mathcal{M}_{X}\right)
$$

for any $\left(X, \mathcal{M}_{X}\right) \in \mathbf{L M S}$ which preserves inverse limits, and to prove that the natural map

$$
\operatorname{Hom}_{\text {LMS }}\left(\left(X, \mathcal{M}_{X}\right), \operatorname{Spec} P\right) \rightarrow \operatorname{Hom}_{\text {Mon }}\left(P, \mathcal{M}_{X}(X)\right)
$$

is bijective.

\section{Acknowledgements}

This research was partially supported by an NSF Postdoctoral Fellowship.

\section{References}

[1] M. Hakim, "Topos Annelés et Schémas Relatifs. Ergebnisse der Mathematik und ihrer Grenzgebiete," SpringerVerlag, Berlin, 1972.

[2] R. Hartshorne, "Algebraic Geometry," Springer-Verlag Berlin, 1977.

[3] H. Becker, Faserprodukt in LRS. http://www.uni-bonn.de/ habecker/Faserprodukt-in-L RS.pdf.

[4] L. Illusie, "Complexe Cotangent et Deformations I. L.N.M. 239," Springer-Verlag, Berlin, 1971.

[5] A. Grothendieck and J. Dieudonné, "Éléments de Géométrie Algébrique," Springer, Berlin, 1960.

[6] J. Giraud, "Cohomologie non Abélienne," Springer, Berlin, 1971.

[7] A. Vistoli, "Notes on Grothendieck Topologies, Fibered Categories, and Descent Theory," Citeseer, Princeton 2004. 